\title{
Angelica gigas Nakai Has Synergetic Effects on Doxorubicin-Induced Apoptosis
}

\author{
Yong-Joon Jeon $\mathbb{D}^{1},{ }^{1}$ Jong-Il Shin $\mathbb{D}^{1},{ }^{1}$ Sol Lee, ${ }^{1}$ Yoon Gyeong Lee, ${ }^{1}$ Ji Beom Kim, \\ Hak Cheol Kwon, ${ }^{2}$ Sung Hun Kim, ${ }^{2}$ Inki Kim, ${ }^{3}$ Kyungho Lee $\mathbb{1},{ }^{1,4}$ and Ye Sun Han ${ }^{5}{ }^{5}$ \\ ${ }^{1}$ Department of Biological Sciences, Konkuk University, Neungdong-ro 120, Gwangjin-gu, Seoul 05029, Republic of Korea \\ ${ }^{2}$ Korea Institute of Science and Technology, Gangneung, Gangwondo 25451, Republic of Korea \\ ${ }^{3}$ Department of Convergence Medicine, Asan Institute for Life Sciences, Asan Medical Center, Seoul 05505, Republic of Korea \\ ${ }^{4}$ Korea Hemp Institute, Konkuk University, Konkuk University, Neungdong-ro 120, Gwangjin-gu, Seoul 05029, Republic of Korea \\ ${ }^{5}$ Department of Advanced Technology Fusion, Konkuk University, Neungdong-ro 120, Gwangjin-gu, Seoul 05029, Republic of Korea
}

Correspondence should be addressed to Kyungho Lee; kyungho@konkuk.ac.kr and Ye Sun Han; yshan@konkuk.ac.kr

Received 7 March 2018; Revised 11 July 2018; Accepted 24 July 2018; Published 1 August 2018

Academic Editor: Claudio Tabolacci

Copyright (C) 2018 Yong-Joon Jeon et al. This is an open access article distributed under the Creative Commons Attribution License, which permits unrestricted use, distribution, and reproduction in any medium, provided the original work is properly cited.

\begin{abstract}
Natural products are valuable sources for drug discovery because they have a wide variety of useful chemical components and biological properties. A quick reevaluation of the potential therapeutic properties of established natural products was made possible by the recent development of the methodology and improvement in the accuracy of an automated high-throughput screening system. In this study, we screened natural product libraries to detect compounds with anticancer effects using HeLa cells. Of the 420 plant extracts screened, the extract of Angelica gigas Nakai (AGN) was the most effective in reducing cell viability of HeLa cells. Markers of apoptosis, such as exposure of phosphatidylserine and cleavage of caspase-7 and PARP, were increased by treatment with the AGN extract. Treatment of the AGN extract increased expression of PKR as well as ATF4 and CHOP, the unfolded protein response genes. In addition, cotreatment of doxorubicin and the AGN extract significantly increased doxorubicininduced apoptosis in HeLa cells. Decursin and decursinol angelate, which were known to have anticancer effects, were the main components of the AGN extract. These results suggest that the extract of AGN containing, decursin and decursinol angelate, increases doxorubicin susceptibility.
\end{abstract}

\section{Introduction}

Doxorubicin (adriamycin), belonging to the anthracycline group, was initially derived from Streptomyces peucetius in the 1960s [1]. Owing to its wide range of anticancer effects against various types of cancers, including solid tumors and hematological malignancies, doxorubicin has occupied an important place in chemotherapy $[2,3]$. The application of doxorubicin, however, has many side effects including cardiac toxicity; therefore, the dose of doxorubicin has been limited [4]. In addition, multidrug resistance or chemoresistance prompted by chemotherapy reduced doxorubicin susceptibility, further limiting its use [5]. Despite these disadvantages, doxorubicin is still an attractive chemotherapeutic drug. To use doxorubicin more efficiently, various therapies have been proposed, including the use of combination therapy as a treatment strategy. Consequently, chemotherapy regimens using doxorubicin, such as FAC (fluorouracil, doxorubicin, and cyclophosphamide), TAC (docetaxel, doxorubicin, and cyclophosphamide), and R-CHOP (rituximab, cyclophosphamide, doxorubicin, vincristine, and prednisone), have been administered to cancer patients $[6,7]$.

Ever since natural products have been recognized as key components of traditional medicines, many drugs and therapies using natural products have been developed $[8$, 9]. Consequently, 24 natural products were developed into approved novel drugs between 1970 and 2006 [10]. However, it is very difficult to select the biologically useful natural products from among the wide diversity of natural products. Although high-throughput screening (HTS) is an efficient method for selecting various natural products, it is known to have drawbacks during natural product screening [11]. In 
this study, we screened natural product libraries via HTS and applied the MTT assay to select the extract of Angelica gigas Nakai (AGN), which exhibited anticancer properties.

The genus Angelica L. belonging to the Umbelliferae family is distributed in Asia, Europe, and North America and comprises more than 60 species $[12,13]$. In China, Japan, and Korea, Angelica L. has been used as a traditional herbal medicine for curing colds, pain, and anemia and has been known as the "female ginseng" due to its beneficial effects on female health $[12,14,15]$. Angelica L. contains a variety of bioactive metabolites, such as pyranocoumarins, essential oils, and polyacetylenes, which exhibit many beneficial effects, including anticancerous, anti-inflammatory, antifungal, antioxidant, and neuroprotective properties [13, 14]. Pyranocoumarins, which are known to be associated with the anticancer effect of Angelica L., are more abundant in the roots of the Angelica gigas Nakai (local name dang-gui in Korea) growing in Korea than in the species growing in China and Japan [16]. Pyranocoumarins are the major components of the alcoholic extract of AGN [17]. Decursin, decursinol angelate, and decursinol are representative pyranocoumarins in the AGN extract. Decursin, the most abundant pyranocoumarin in the AGN extract, has been reported to show anticancer effects in various cancer cells $[13,14]$. In addition, the alleviation of neurotoxicity and nephrotoxicity via its antioxidant properties is the other beneficial effect of decursin $[18,19]$. As neurotoxicity and nephrotoxicity are some of the side effects of doxorubicin [20], the combination of doxorubicin and the AGN extract could offer a strategy for increasing the effectiveness of doxorubicin.

Integrated stress response (ISR) is a cellular stress response induced by various stress stimuli, leading to the phosphorylation of eukaryotic translation initiation factor 2 alpha $(\operatorname{eIF} 2 \alpha)$. The phosphorylation of eIF $2 \alpha$ is mediated by four kinases, General Control Nondepressible protein 2 (GCN2), Protein Kinase R (PKR), PKR-like ER localized eIF2 $\alpha$ Kinase (PERK), and Heme-Regulated Inhibitor kinase (HRI) [21, 22]. Although each kinase recognizes different stimuli, ISR is initiated by very diverse stress stimuli via the four kinases and prompts a cellular response for determining cell fate. The upregulation of eIF $2 \alpha$ phosphorylation attenuates global protein translation to reduce cellular stress [21]. However, the rates of translation of mRNAs including the second $5^{\prime}$-uORF such as activating transcription factor 4 (ATF4) are further increased by eIF $2 \alpha$ phosphorylation [23]. ATF4 activates the expression of genes that regulate cellular homeostasis in order to protect cells or increases the expression of downstream transcription factors such as $\mathrm{C} / \mathrm{EBP}-$ homologous protein (CHOP) [24]. In conditions of severe stress, ATF4 and CHOP induce cell death by activating downstream death factors or by producing ROS via increased protein synthesis $[24,25]$. Therefore, ISR is an important mechanism for determining cell fate by inducing a cellular response by various cellular stimuli via the eIF2 $\alpha$-ATF4 pathway.

In this study, the AGN extract effectively induced apoptosis in HeLa cells. As the cell death induced by doxorubicin was related to eIF $2 \alpha$ phosphorylation, we investigated the synergetic effect between doxorubicin and the AGN extract.
The administration of the AGN extract enhanced ATF4 and CHOP expression in doxorubicin-treated HeLa cells, resulting in an increase in doxorubicin-induced apoptosis.

\section{Materials and Methods}

Doxorubicin was purchased from Sigma-Aldrich (St. Louis, $\mathrm{MO})$. The PKR inhibitor $\mathrm{C} 16$ was purchased from Calbiochem (La Jolla, CA). The anti-phospho eIF2 $\alpha$, antieIF2 $\alpha$, and anti-CHOP antibodies were purchased from Cell Signaling Technology (Beverly, MA). The anti-GAPDH, antiactin, anti-caspase-3, anti-PKR, anti-Bcl-2, and anti-PARP antibodies were purchased from Santa Cruz Biotechnology (Santa Cruz, CA). The annexin V probe was purchased from Bioacts (Korea). Hoechst 33342 was purchased from Invitrogen (Carlsbad, CA)

2.1. Cell Culture. The HeLa cell line was obtained from the Korean Cell Line Bank (KCLB). The HeLa cells were cultured in Dulbecco's modified Eagle's media (Welgene, Daegu, Korea) supplemented with $10 \%$ heat-activated fetal bovine serum (Biowest, Nuaillé, France) and 1\% penicillin or streptomycin mixtures (GIBCO, ThermoFisher, MA, USA). The HeLa cells were incubated in a humidified atmosphere at a $\mathrm{CO}_{2}$ concentration of $5 \%$ and a temperature of $37^{\circ} \mathrm{C}$.

2.2. Cell Viability Assay. The cells were seeded in 48-well plates and incubated for $16 \mathrm{~h}$. The cells were treated with different concentrations of doxorubicin for $24 \mathrm{~h}$. Cell survival was measured using the MTT [3-(4, 5-dimethylthiazol-2yl)-2, 5-diphenyltetrazolium bromide] assay (Sigma-Aldrich, ST Louis, MO). In brief, PBS containing $5 \mathrm{mg} / \mathrm{ml}$ MTT was diluted with the media at a concentration of $0.5 \mathrm{mg} / \mathrm{ml}$ and incubated in a humidified chamber containing $\mathrm{CO}_{2}$ for $2 \mathrm{~h}$. The medium was aspirated from each well and $200 \mu \mathrm{l}$ DMSO was added to dissolve the Formazan crystals. The absorbance of each well was measured using a UVM 340 plate reader at a wavelength of $570 \mathrm{~nm}$.

2.3. Immunoblot Analyses. The cells were harvested using RIPA lysis buffer [containing $150 \mathrm{mM} \mathrm{NaCl}, 1 \%$ Triton X-100, $1 \%$ sodium deoxycholate, $0.1 \% \mathrm{SDS}, 50 \mathrm{mM}$ Tris- $\mathrm{HCl}$, and $2 \mathrm{mM}$ EDTA] along with $1 \%$ phosphatase inhibitor and protease inhibitor cocktail (Roche Diagnostics, Germany). The protein concentration was quantified using the Pierce BCA protein assay kit (Thermo Scientific, Australia). Proteins boiled in $1 \mathrm{x}$ sample buffer [containing $500 \mathrm{mM}$ Tris- $\mathrm{HCl}$ (pH 6.8), 10\% SDS, 20\% glycerol, 0.05\% bromophenol blue, and $1 \% \beta$-mercaptoethanol] for 5 minutes at $100^{\circ} \mathrm{C}$ were separated on SDS-polyacrylamide gels. The proteins were electrotransferred to Immobilon-P membranes (Millipore, Temecula, CA) and blotted with the indicated antibodies at $4^{\circ} \mathrm{C}$ overnight in Tris-Buffered saline containing $0.08 \%$ Tween 20 (TBST) and $1 \%$ nonfat milk. The membranes were then incubated with horseradish peroxidase-conjugated antibodies at room temperature for $2 \mathrm{~h}$, and the band signal was detected using a LAS-3000 Luminescent Image Analyzer (Fujifilm, Japan). To determine the equal loading of samples, the blots were stripped in stripping buffer [containing 
TABLE 1: EC50 values of the natural products.

\begin{tabular}{lllll}
\hline Natural product extract & $\# 478$ & $\# 622$ & \#1114 & \\
\hline EC 50 value & 46.77 & 10.36 & 29.12 & 43.26 \\
\hline
\end{tabular}

$100 \mathrm{mM} \beta$-mercaptoethanol, $2 \%$ SDS, and $62.5 \mathrm{mM}$ Tris- $\mathrm{HCl}$ ( $\mathrm{pH} 6.8$ )] at $50^{\circ} \mathrm{C}$ for 20 minutes, followed by washing twice with TBST buffer for 15 minutes each time, and reprobed with an antibody specific for $\beta$-actin or GAPDH.

2.4. Measurement of Apoptosis. The cells were cultured in a confocal dish and treated with doxorubicin and/or the AGN extract for $24 \mathrm{~h}$. The cells were washed with PBS and binding buffer (20 mM Hepes at pH 7.4, $150 \mathrm{mM} \mathrm{NaCl}$, and $2.5 \mathrm{mM}$ $\mathrm{CaCl}_{2}$ ). The staining solution was prepared by diluting the annexin $\mathrm{V}$ probe and Hoechst 33342 with the binding buffer at concentration ratios of 1:200 and 1:5000, respectively. The cells were stained with the staining solution for 20 minutes. The stained cells were observed using a confocal microscope (Zeiss LSM 800, Carl Zeiss).

2.5. Preparation of Crude Extract. The crude extract samples used in this study were provided by Natural Product Library of Korea Institute of Science and Technology, Gangneung Institute, Gangneung, Korea. The natural product library was made from Korean native plants. The preparation of A. gigas extract is as follows. The roots of A. gigas were purchased in a local oriental medicine market in Bonghwa, Korea, in 2015. The plant materials were authenticated by Professor DS Jang at College of Korean Medicine at Kyung Hee University. The specimen was deposited in KIST Natural Product Library (Deposit number: \#BS0622A1). The dried materials (100 g) were cut and extracted twice with $1 \mathrm{~L}$ of ethanol by reflux at $60^{\circ} \mathrm{C}$ for 2 hours. Thereafter, the extract was filtered and concentrated using a rotary evaporator under vacuum at $35^{\circ} \mathrm{C}$.

2.6. Chemical Composition of the AGN Extract. To investigate the chemical constituents of the AGN extract, LC/MS analyses were performed on an Agilent 1200 HPLC system equipped with UV and ESI-MS detection, using a Phenomenex Luna C18 column $(150 \times 4.6 \mathrm{~mm}, 5 \mu \mathrm{m})$. The mobile phase used was a linear gradient of $10-100 \%$ acetonitrile in water (containing $0.05 \%$ formic acid) for over 30 minutes at a flow rate of $0.7 \mathrm{ml} / \mathrm{min}$. The HPLC chromatogram was monitored at a UV wavelength of $254 \mathrm{~nm}$. Mass analysis was performed using the positive-ion mode. After analyses, the peaks in the HPLC chromatogram were identified by comparing the obtained UV spectra and mass spectra with those of compounds previously reported from A. gigas.

2.7. Quantitative Real-Time PCR. Quantitative real-time PCR was accomplished with HiPi Real-Time PCR $2 \times$ Master Mix SYBR Green (ELPiS Biotechnology, Korea) with 40 cycles. The cycle threshold (cT) was observed in extension step and used for calculation of relative gene expression. Analysis of melting curve was carried out in order to convict specific amplification.
2.8. Statistical Analyses. The values in the figures are expressed as the mean $\pm \mathrm{SD}$. The figures in this study represent the results of experiments performed more than three times. Statistical analyses of the data obtained from the control and the treated groups were performed by using Student's t-test. Values of $\mathrm{P}<0.05$ indicate statistical significance.

\section{Results}

3.1. Screening of the Most Effective Anticancer Candidate from the KIST Natural Product Library. Initially, natural product extract libraries were selected to obtain components with anti-inflammatory effects. Since recent studies have shown that cancer and inflammation are closely related [26], in this study we investigated the anticancer effects of natural product extracts from the KIST Natural Product Library. To select extracts with anticancer properties from among approximately 420 natural products, HeLa cells were treated with 50 $\mu \mathrm{g} / \mathrm{ml}$ of each natural product extract for 24 hours and natural product extracts that markedly reduced cell viability to below $50 \%$ were identified. Through this process, four extracts, \#BE0478A1, \#BE0622A1, \#BE1114A1, and \#BE1197A1, were selected. In order to compare the anticancer effects of the four extracts with EC50 value, HeLa cells were treated with various concentrations of each extract for 24 hours and EC50 value of the four natural product extracts was calculated (Table 1 and Figure 1(a)). The results showed that the \#BE0622A1 extract was the most efficient in reducing HeLa cell viability, depending on the concentration (Figure $1(\mathrm{a})$ ), and the $\mathrm{EC}_{50}$ value was the lowest compared to that of the other extracts (Table 1). Apoptosis has been recognized as an important mechanism for cancer therapy, and many anticancer drugs are known to induce apoptosis in cancer cells [27]. The activation of caspase-7 (cCas-7) and the cleavage of PARP are representative markers of apoptosis. Thus, we compared the apoptosis induced by the extracts by observing cCas7 activation and PARP cleavage (Figure 1(b)). Similarly to the cell viability, cCas-7 activation and PARP cleavage were enhanced more after treatment with the \#BE0622A1 extract than with extracts \#BE1114A1 and \#BE1197A1.

The apoptosis mediated by the extract \#BE0622A1 was also confirmed by annexin $\mathrm{V}$ staining (Figure 1(c)). Among the natural products evaluated, \#BE0622A1 proved to be the most effective anticancer extract in HeLa cells. The extract \#BE0622A1 was prepared from root of Angelica gigas Nakai (AGN).

3.2. Activation of ISR by Treatment with the Angelica gigas Nakai Extract. ISR has been known to cope with diverse stresses, resulting in cell death or adaptation [21]. Four kinases sensing various stress stimuli phosphorylate eIF $2 \alpha$ to initiate ISR. As the AGN extract is a crude mixture, we speculated that the AGN extract can offer numerous kinds of 


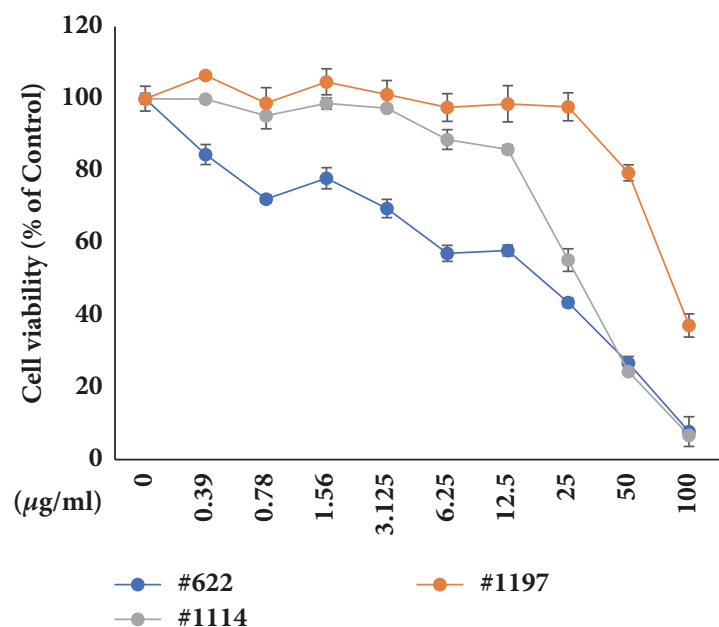

(a)

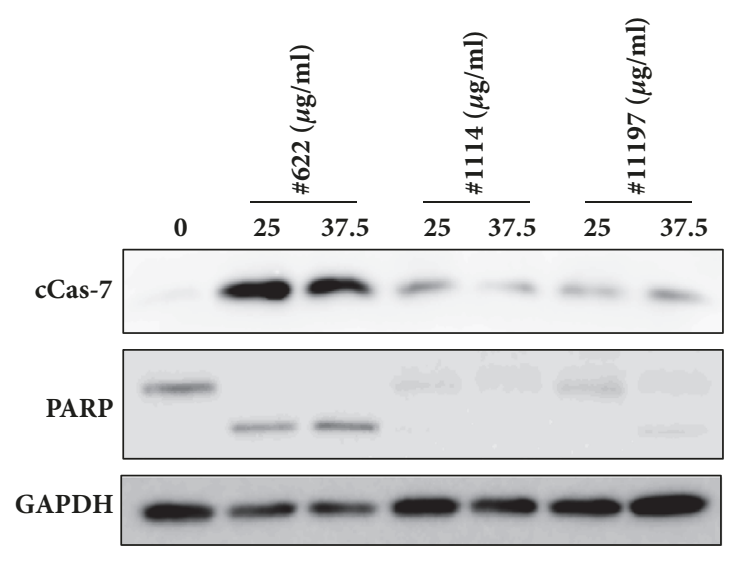

(b)

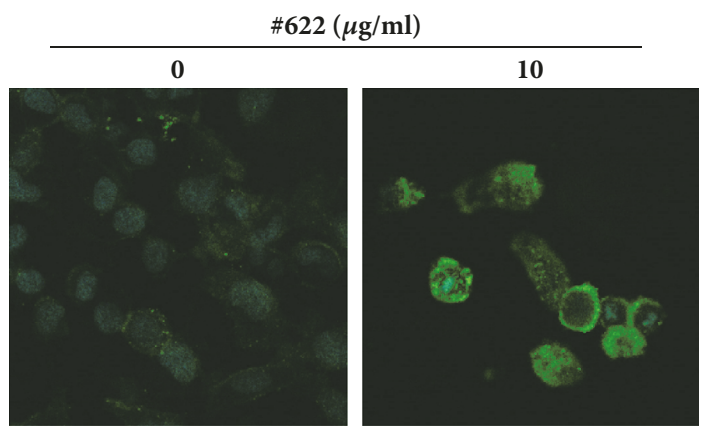

(c)

Figure 1: Angelica gigas Nakai (\#BE0622A1), one of the 420 natural products, showed the most effective anticancer effect. (a) The HeLa cells were treated with various concentrations of the indicated natural products for $24 \mathrm{~h}$, and the MTT assay was subsequently performed. (b) The HeLa cells were treated with various concentrations of \#BE0622A1, \#BE1114A1, and \#BE1197A1 for $24 \mathrm{~h}$, and cell lysates were subjected to immunoblot analyses using specific antibodies for cleaved form of caspase-7, PARP, and GAPDH. (c) The HeLa cells were treated with various concentrations of \#BE0622A1 for $24 \mathrm{~h}$ and apoptosis was analyzed by annexin V staining with FITC conjugation.

stimuli to HeLa cells. Accordingly, we investigated whether the AGN extract could increase phosphorylation of eIF $2 \alpha$. The $\mathrm{EC}_{50}$ value of the AGN extract was approximately 10 $\mu \mathrm{g} / \mathrm{ml}$ (Figure 1(a)). Based on these results, the level of eIF $2 \alpha$ phosphorylation was measured after treatment with the AGN extract for $16 \mathrm{~h}$ (Figure 2(a)). The level of eIF2 $\alpha$ phosphorylation was not affected by concentration of the AGN extract. However, the expression of ATF4 and CHOP, downstream factors of eIF $2 \alpha$, was enhanced by treatment with the AGN extract. Phosphorylation of eIF2 $\alpha$ was increased in a timedependent manner (Figure 2(b)). The phosphorylation of eIF $2 \alpha$ was increased after $2 \mathrm{~h}$ treatment with the AGN extract. At the same time, the expression of PKR, one of the eIF $2 \alpha$ kinases, was also increased. The transcription of ATF4 and CHOP was increased in a time-dependent manner, and expression of ATF4 and CHOP was also increased sequentially after $4 \mathrm{~h}$ and $8 \mathrm{~h}$, respectively (Figures 2(b)-2(d)). We therefore concluded that the AGN extract-mediated apoptosis was associated with ISR via the eIF $2 \alpha$-ATF4-CHOP pathway. Treatment of the AGN extract also increased the splicing of XBP1 mRNA, suggesting activation of the IRE1 $\alpha$ pathway (data not shown).
3.3. The AGN Extract Showed a Synergetic Effect on the Doxorubicin-Induced Apoptosis. Previous studies have demonstrated that phosphorylation of eIF $2 \alpha$ improved doxorubicin-mediated cell death in cancer cells $[28,29]$. In addition, combination therapy has been widely used as a method for overcoming the limitations of chemotherapy in the treatment of cancer [30]. Therefore, we hypothesized that doxorubicin and the AGN extract could exhibit a synergetic effect based on eIF $2 \alpha$ phosphorylation. To investigate whether AGN affects doxorubicin-induced cell death, doxorubicin was coadministered with the AGN extract for $24 \mathrm{~h}$ (Figure 3(a)). Although cotreatment with $0.5 \mu \mathrm{g} / \mathrm{ml}$ AGN extract and various concentration of doxorubicin hardly affected doxorubicin-induced cell viability, cotreatment with $1 \mu \mathrm{g} / \mathrm{ml} \mathrm{AGN} \mathrm{extract}$ and 1 $\mu \mathrm{M}$ doxorubicin significantly reduced the cell viability. We then examined the effect of the AGN extract on doxorubicininduced apoptosis through cCas-7 and PARP. Cotreatment with $1 \mu \mathrm{M}$ doxorubicin with $1 \mu \mathrm{g} / \mathrm{ml}$ or $2 \mu \mathrm{g} / \mathrm{ml} \mathrm{AGN} \mathrm{extract}$ markedly increased the activation of caspase- 7 and the cleaved form of PARP (Figure 3(b)), which means that the doxorubicin-mediated apoptosis was greatly enhanced by the administration of the AGN extract. Furthermore, the 


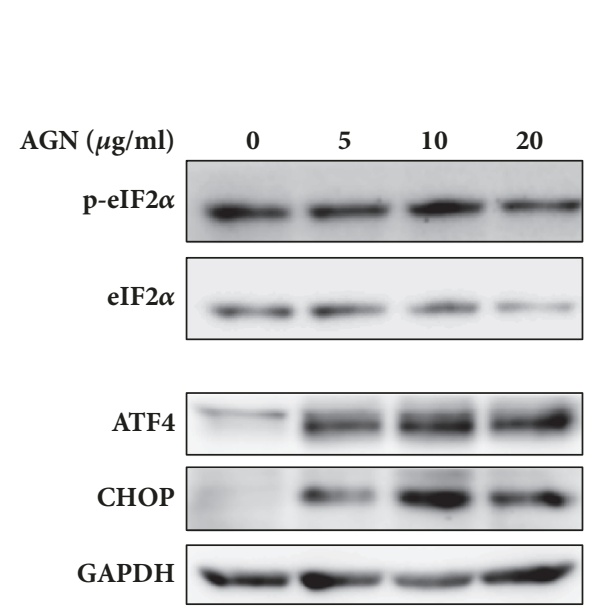

(a)

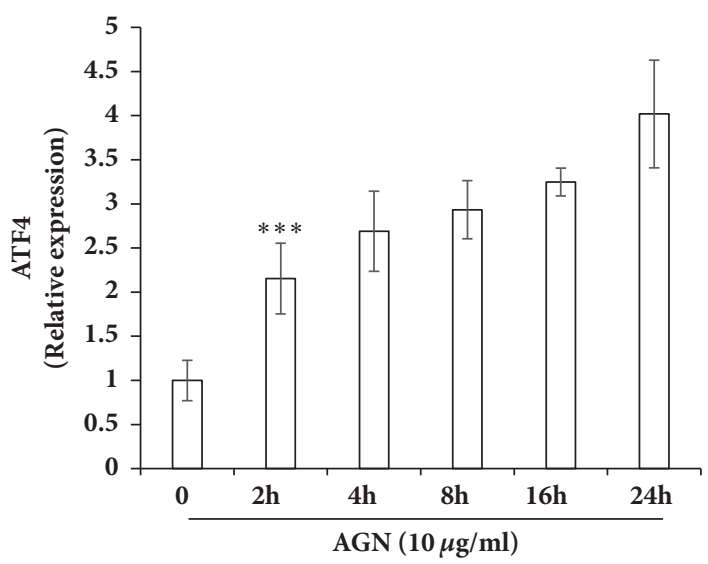

(c)

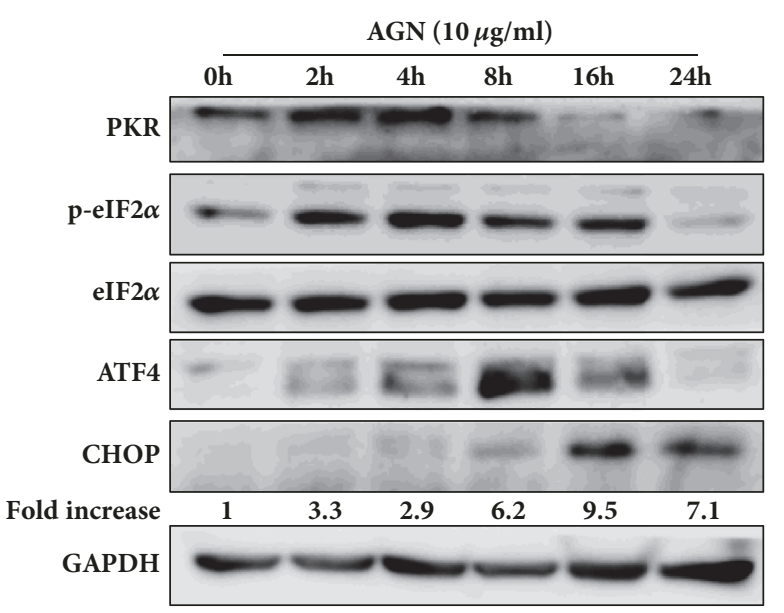

(b)

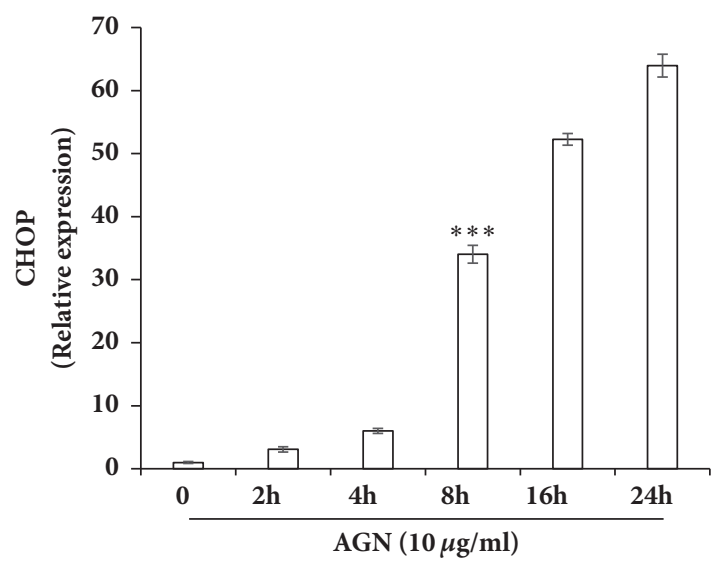

(d)

FIGURE 2: The AGN extract activated the integrated stress response (ISR) in HeLa cells. The HeLa cells were treated with the indicated concentrations of the AGN extract for $16 \mathrm{~h}$ (a) or with $10 \mu \mathrm{g} / \mathrm{ml}$ of the AGN extract for the indicated time periods (b). Cell lysates were subjected to immunoblot analyses using the indicated antibodies. The indicated fold increase in CHOP expression is the ratio of the CHOP to GAPDH. (c, d) The mRNA levels of ATF4 and CHOP were determined by real-time quantitative PCR. Statistical significance of the difference as calculated by Student's $t$-test is with $* * * \mathrm{p}<0.001$.

administration of the AGN extract along with doxorubicin considerably increased the cleaved form of caspase- 8 in contrast to the administration of doxorubicin alone (Figure 3(b)). The upregulation of the cleaved form of caspase- 8 indicates that the receptor-mediated/extrinsic apoptotic pathway is activated [31]. The fluorescence intensity of the apoptotic marker as indicated by annexin V staining in the Hoechststained cells was also stronger when the cells were cotreated with doxorubicin and the AGN extract than with doxorubicin alone (Figure 3(c)). These results suggest that doxorubicininduced apoptosis was enhanced via the extrinsic apoptotic pathway by the administration of the AGN extract.

\subsection{Upregulation of Apoptosis via the ATF4-CHOP Pathway.} The abovementioned results demonstrate that the AGN extract has a synergetic effect on doxorubicin-induced apoptosis. We further speculated whether ISR is correlated to the AGN extract-mediated synergy. To confirm the correlation, HeLa cells were cotreated with doxorubicin and the AGN extract (Figure 4). Figures 4(a)-4(c) represent the timedependent changes in transcriptional and translational level expression of ATF4 and CHOP at the indicated concentrations, and Figure 4(d) depicts the changes at various concentrations of doxorubicin and the AGN extract over 24 hours. Although the treatment with doxorubicin alone hardly enhanced the expression of ATF4 and CHOP, cotreatment with the AGN extract and doxorubicin increased the expression of ATF4 and CHOP. However, in comparison to the administration of the AGN extract alone, the expression level of ATF4 and CHOP was low by cotreatment (Figures $4(\mathrm{a})$ and 4(d)). The expression of death receptor 5 (DR5), which is a downstream factor of CHOP and related to caspase- 8 activation, was not changed in correspondence with the expression of $\mathrm{CHOP}$ (Figure 4). The expression of DR5, therefore, was not related to the ATF4-CHOP pathway. Nevertheless, these results suggest that the synergetic effect of the AGN extract is related to the upregulation of ATF4 and CHOP expression. 


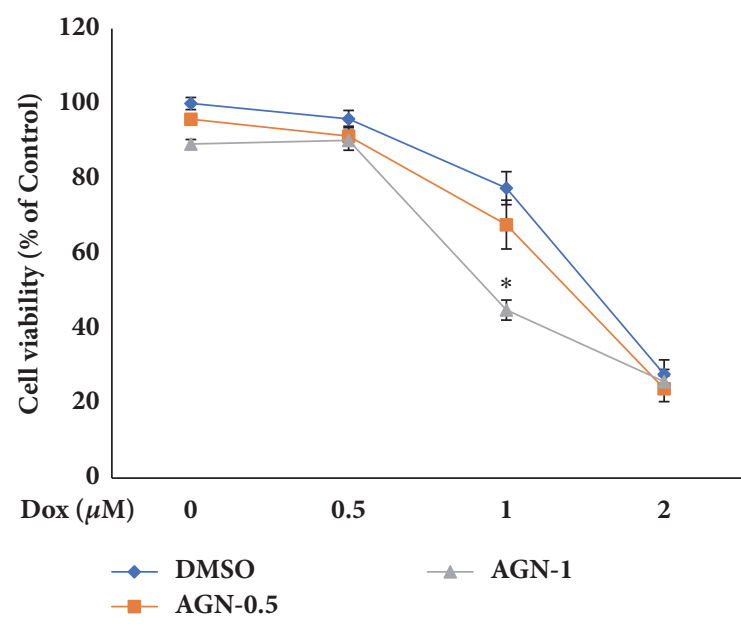

(a)

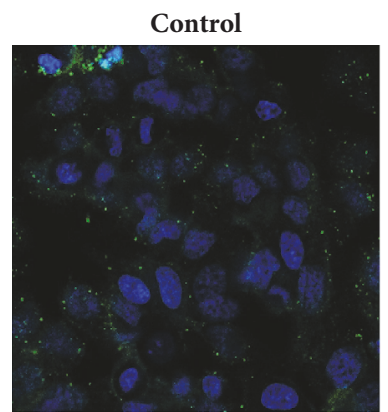

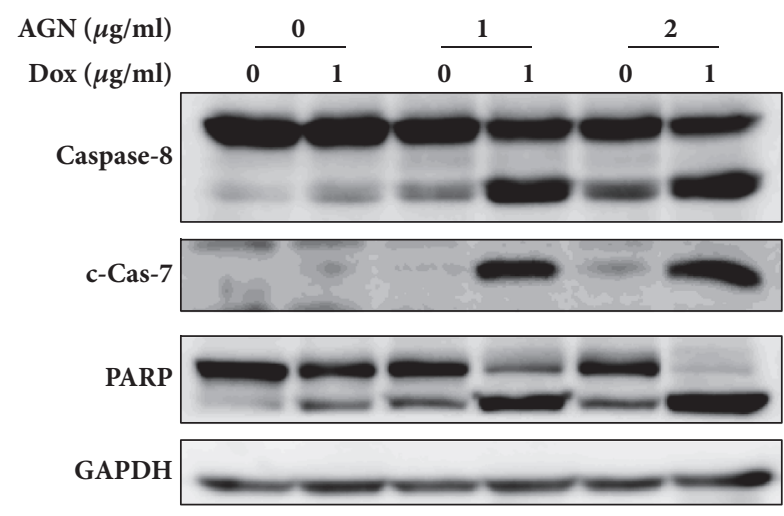

(b)
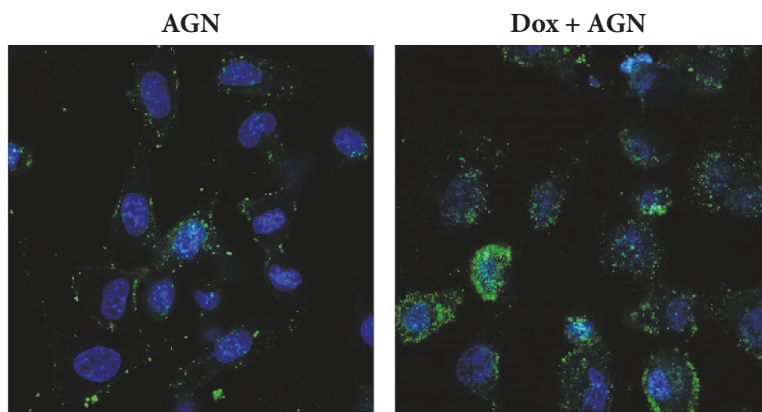

(c)

FIgURE 3: Coadministration of the AGN extract with doxorubicin enhanced doxorubicin-induced apoptosis in HeLa cells. (a) The cells were cotreated with the indicated concentrations of doxorubicin and the AGN extract for $24 \mathrm{~h}$, and cell viability was measured using the MTT assay. The statistical significance of the differences, as calculated by Student's t-test, was determined with $* \mathrm{p}<0.01$. (b) The cells were cotreated with the indicated concentrations of doxorubicin and the AGN extract for $24 \mathrm{~h}$ and subjected to immunoblot analyses using specific antibodies as indicated. (c) The cells were cotreated with $1 \mu \mathrm{M}$ doxorubicin and $1 \mu \mathrm{g} / \mathrm{ml} \mathrm{AGN} \mathrm{extract} \mathrm{for} 16 \mathrm{~h}$ and apoptosis was analyzed by costaining with annexin V-FITC and Hoechst probes.

3.5. Chemical Composition of the AGN Extract. Sowndhararajan et al. reported that there are differences in major components depending on the plant part, yield, and extraction method in essential oil of various species of Angelica [32]. Therefore, to clarify the major components and biologically active components of the AGN extract, the AGN extract was divided into eight fractions. When HeLa cells were treated with various concentrations of each fraction for 24 hours, fraction \#3 reduced HeLa cell viability most effectively (Supplementary Materials, Figure S1a). Also, fraction \#3 not only activated caspase 7 but also increased expression of CHOP in HeLa cells (Supplementary Materials, Figure S1b). We then checked the HPLC-MS chromatogram and the $1^{\mathrm{H}}$ NMR spectrum of each fraction to find major components of each fraction. Figure 5(b) revealed that fraction \#3 of the AGN extract contained three coumarins, 7-demethylsuberosin $(\mathrm{m} / z 231)$, decursin $(\mathrm{m} / z$ 329), and decursinol angelate $(\mathrm{m} / z$ 329). Among these substances, decursin and decursinol angelate were the main constituents (Figure 5(b)). These compounds are known as the principal constituents of $A$. gigas, which have significant anticancer effects in various cancer models $[8,9]$. Therefore, the results proposed that these compounds play an important role in induction of apoptosis in HeLa cells.

\section{Discussion}

Although doxorubicin is an efficient anticancer drug, various side effects such as drug resistance and cytotoxicity have limited the use of doxorubicin. Many attempts have been made to overcome the limitations of doxorubicin usage and enhance its efficiency, and combination therapy has frequently been used as a strategy for the efficient use of doxorubicin. In the present study, the combination of doxorubicin and the AGN extract markedly enhanced doxorubicin-induced apoptosis in HeLa cells (Figure 3). This event was associated with the AGN extract-mediated expression of ATF4 and CHOP (Figures 2 and 4). Particularly, unlike in HeLa cells, cotreatment of doxorubicin and the AGN extract did not significantly increase doxorubicin-induced apoptosis in wild type WI-38 cells (Supplementary Materials, Figure S2). These results demonstrate that administration of the AGN extract 


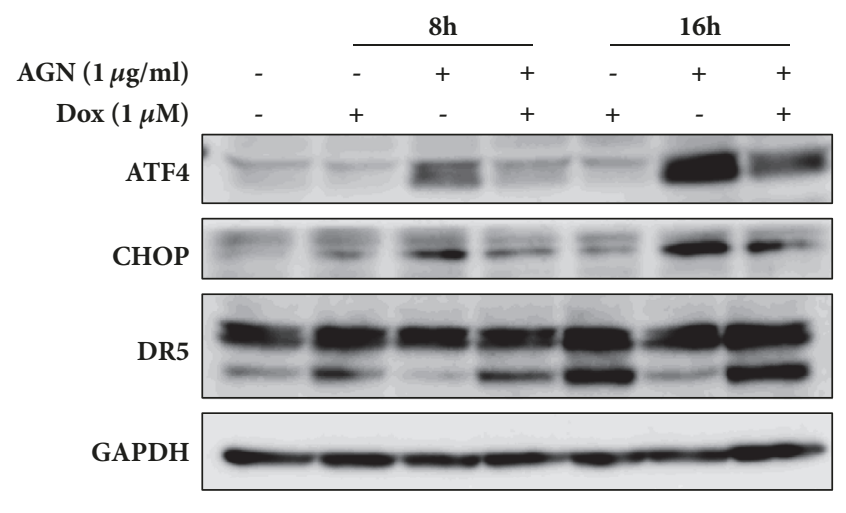

(a)

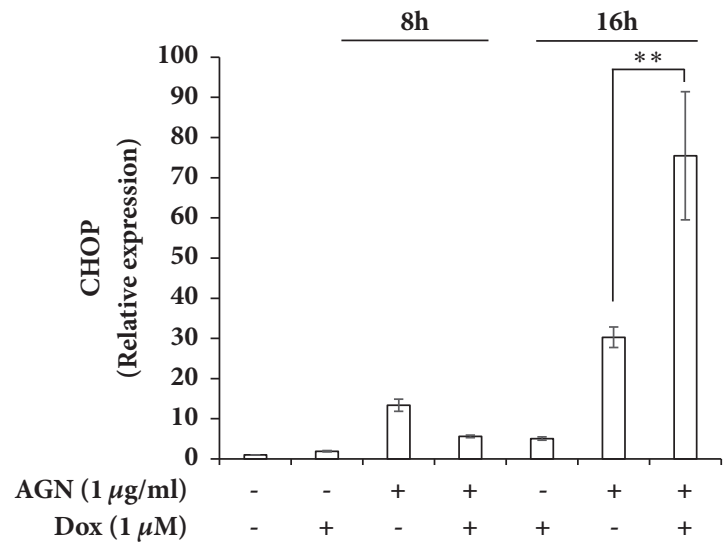

(c)

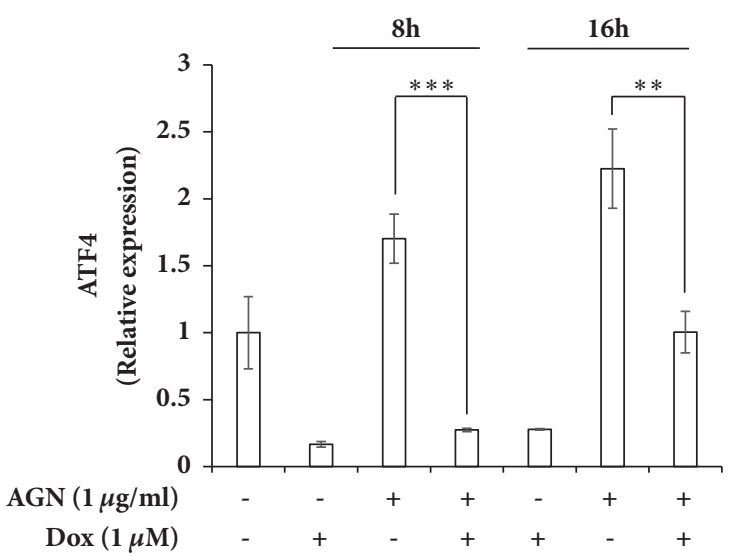

(b)

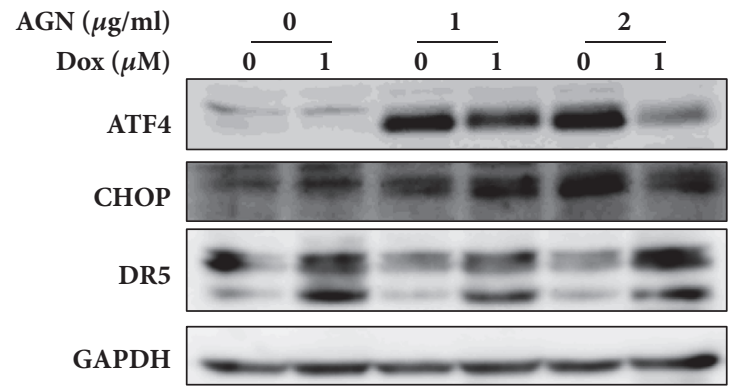

(d)

Figure 4: Administration of the AGN extract activated the ATF4-CHOP pathway in doxorubicin-treated HeLa cells. (a) The cells were cotreated with $1 \mu \mathrm{M}$ doxorubicin and $1 \mu \mathrm{g} / \mathrm{ml} \mathrm{AGN}$ extract for the indicated time periods and immunoblot analyses were performed using the indicated antibodies. (b, c) The mRNA level of ATF4 and CHOP were determined by real-time quantitative PCR. Statistical significance of the difference as calculated by Student's $t$-test is with $* * \mathrm{p}<0.01$ or $* * * \mathrm{p}<0.001$. (d) The cells were cotreated with $1 \mu \mathrm{M}$ doxorubicin and $1-2$ $\mu \mathrm{g} / \mathrm{ml}$ AGN extract for $24 \mathrm{~h}$ as indicated and immunoblot analyses were performed using the indicated antibodies.

increased the efficiency of doxorubicin in HeLa cells through the activation of ISR, suggesting that the AGN extract works synergistically with doxorubicin.

The AGN extract was the most effective among the natural products screened in inducing apoptosis in HeLa cells (Figure 1). AGN-mediated apoptosis was associated with the eIF2 $\alpha$-ATF4-CHOP pathway (Figure 2). Accordingly, we investigated the activity of eIF $2 \alpha$ kinases PERK and PKR under conditions that the cells were treated with the AGN extract alone. PERK, which is known to be activated by ER stress [21], was not activated by treatment with 10 $\mu \mathrm{g} / \mathrm{ml}$ AGN extract (data not shown). On the other hand, the AGN extract increased the expression of PKR, which corresponded to the time when eIF $2 \alpha$ phosphorylation is increased (Figure 2(b)). Treatment with the PKR inhibitor C16 significantly inhibited the activation of cCas-7 and restored cell viability that had been reduced by treatment with the AGN extract (Supplementary Materials, Figures S3a and $\mathrm{S} 3 \mathrm{~b})$. These data show that the AGN extract seems to induce apoptosis by activating PKR in HeLa cells. Treatment with C16, however, neither inhibited nor increased the expression of ATF4 and CHOP (Supplementary Materials, Figure S3c). Previous studies reported that the inhibition of PKR using C16 decreased caspase- 3 activation by inhibiting NF- $\kappa \mathrm{B}-$ induced inflammation and FADD phosphorylation $[33,34]$. p53 is another PKR-mediated apoptotic signal transducer [29, 35]. Therefore, it seems that there is no correlation between the activity of PKR and expression of ATF4-CHOP in AGN extract-treated HeLa cells; upregulation of the eIF2 $\alpha$-ATF4CHOP pathway by treatment with $\mathrm{C} 16$ shows the possibility of correlation to another eIF $2 \alpha$ kinase.

Expression of ATF4 and CHOP was not induced by treatment of doxorubicin alone in HeLa cells, but coadministration with the AGN extract markedly increased the expression of both genes. Indeed, the expression level of ATF4 and CHOP was higher when the cells were treated with the AGN extract alone than with cotreatment (Figure 4). Treatment of breast cancer cells with doxorubicin effectively increases the phosphorylation of eIF $2 \alpha$ but suppresses the expression of ATF4 at the transcription level $[28,36]$. Nevertheless, administration of the AGN extract increased the expression of ATF4 and CHOP in doxorubicin-treated HeLa 
<smiles>CC(C)=CCc1cc2ccc(=O)oc2cc1O</smiles>

(1)

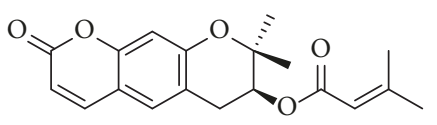

(2)

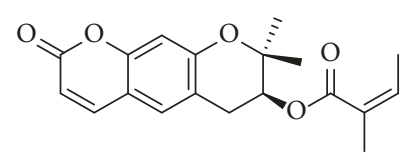

(3)

(a)

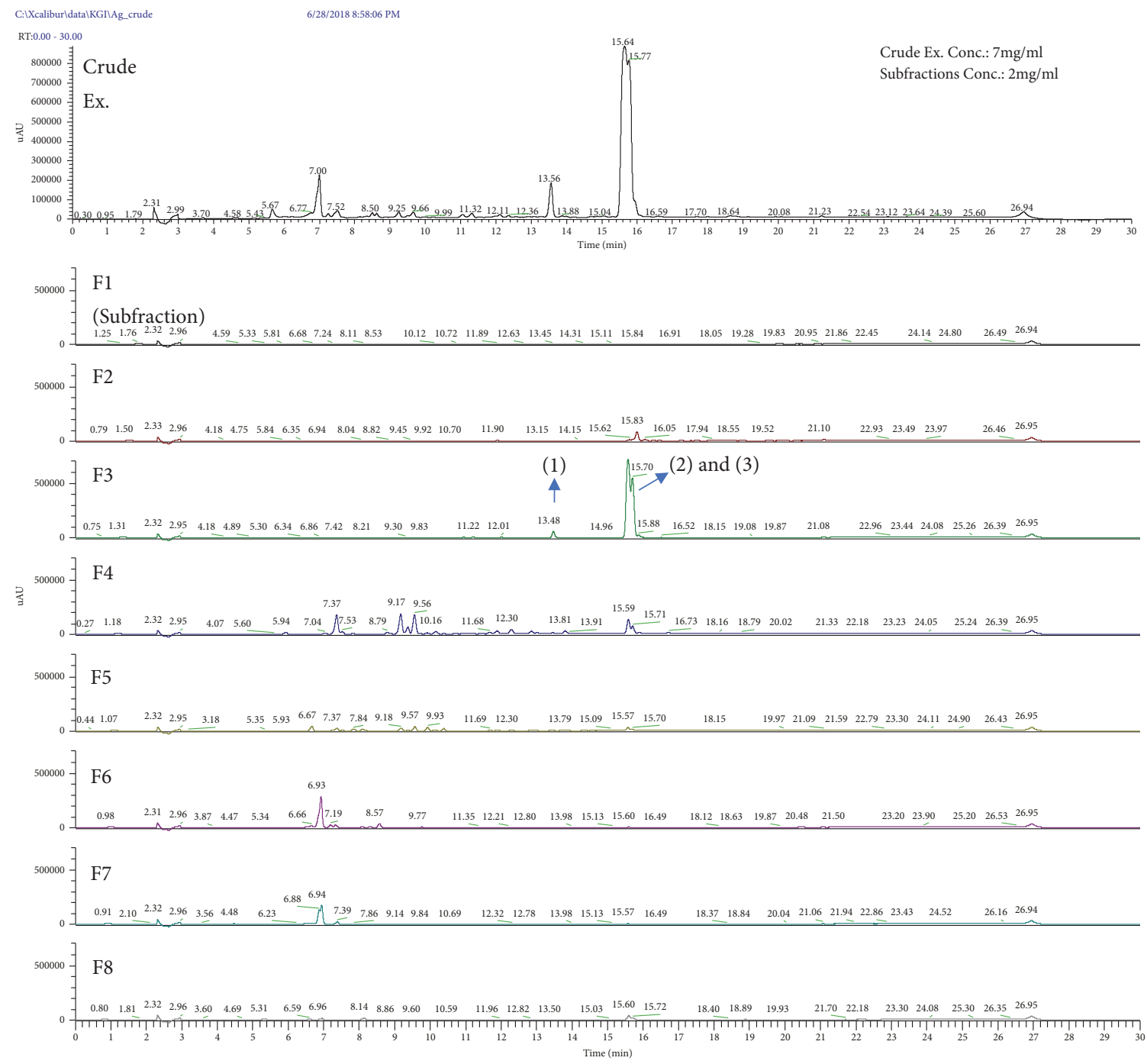

(b)

FIgURE 5: Chemical composition of the AGN extract. (a) Chemical structures of the three components: (1) 7-demethylsuberosin, (2) decursin, and (3) decursinol angelate. (b) HPLC-MS chromatograms of the AGN extract and the fractions.

cells, indicating that ATF4 and CHOP play a role in the synergetic effect of doxorubicin and the AGN extract. Both ATF4 and CHOP are recognized as key transcription factors functioning downstream of eIF $2 \alpha$, which regulate the expression of genes associated with cellular homeostasis and cell death $[24,37]$. Although many studies indicate that ATF4 plays a role in cellular protection in association with the expression of redox enzymes, autophagy, translation, and multidrug-resistant gene expression [24], ATF4 promotes proapoptotic factors such as Puma and Noxa [38, 39]. Therefore, it is likely that increased expression of ATF4 by the AGN extract enhanced doxorubicin-induced apoptosis in HeLa cells. Indeed, an increase in protein synthesis by ATF 4 expression induced ROS-mediated apoptosis [25]. However, treatment with $\mathrm{N}$-acetyl-cystein (NAC), which is a precursor of glutathione, did not affect the cell viability in HeLa cells cotreatment with doxorubicin and the AGN extract (data not shown).

$\mathrm{CHOP}$ is a transcription factor functioning downstream of ATF4 and is a well-known death factor in ISR. CHOPmediated apoptosis is associated with several apoptotic factors including the anti- and proapoptotic Bcl-2 families, 
microRNAs, TRB3, DR5, and GADD34 [40, 41]. DR5 is known to induce apoptosis by activating caspase-8 [42]. In fact, cotreatment with doxorubicin and the AGN extract significantly increased the activation of caspase-8 (Figure 3 ). However, administration of the AGN extract did not enhance doxorubicin-mediated DR5 expression (Figure 4). Although the increase in caspase- 8 activation by the AGN extract was not related to the expression of CHOP-DR5, the AGN extract is known to activate caspase- 8 by enhancing the expression of the DR5 ligand TRAIL [43]. Accordingly, it is possible that upregulation of caspase- 8 activation was caused by the expression of TRAIL in AGN extract-treated cells. Inhibition of the anti-apoptotic protein $\mathrm{Bcl}-2$ and activation of the proapoptotic protein Bax/Bak are known as CHOPmediated apoptotic mechanisms [44, 45]. Treatment with the ANG extract resulted in downregulation of Bcl-2 and upregulation of Bax expression [46]. Also, decursin and decursinol angelate, which are major components of the AGN extract, effectively decreased $\mathrm{Bcl}-2$ expression [43]. Therefore, there is a possibility that CHOP functions as an apoptotic factor in AGN extract-treated cells. However, knockdown of CHOP using specific shRNA did not affect the apoptosis in AGN extract-treated HeLa cells even in cotreated conditions with doxorubicin (Supplementary Materials, Figure S4). Therefore, as mentioned above, CHOP plays an important role in ISR-mediated apoptosis but does not seem to affect AGN extract-mediated apoptosis.

Many studies have shown that decursin and decursinol angelate are major compounds of Angelica gigas Nakai, and they are known to have primary responsibility for the anticancer effect of Angelica gigas Nakai [13, 14, 46]. Therefore, we also analyzed the composition of the AGN extract and found that decursin and decursinol angelate are major components of the AGN extract (Figure 5). Previous studies have shown that decursin has synergetic effects with doxorubicin [47, 48]. Decursin enhanced caspase-9-mediated apoptosis in doxorubicin-treated multiple myeloma cells, via the mTOR and STAT3 pathways [47] and other reports showed that decursin increased caspase-8-mediated apoptosis by increasing TRAIL sensitivity [49]. Thus, the apoptotic pathway induced by decursin might activate different pathways, depending on the cell's characteristics and conditions. Further studies to characterize the relationship between decursin and doxorubicin are needed. Decursin inhibits the expression of P-glycoprotein, which is an efflux pump that reduces the efficiency of doxorubicin by lowering its cellular concentration [48]. Decursin is also known to inhibit cancer cell metastasis and angiogenesis $[50,51]$. Therefore, it could be used as an efficient component in combination therapies along with several other anticancer drugs, including doxorubicin.

\section{Conclusions}

Collectively, our results showed that the AGN extract induced expression of PKR, ATF4, and CHOP as well as phosphorylation of eIF $2 \alpha$. It significantly increased apoptosis and enhanced doxorubicin susceptibility in HeLa cells. We also analyzed the composition of the AGN extract and found that decursin and decursinol angelate were the main components of the extract. Consequently, the AGN extract comprising decursin and decursinol angelate could be an effective material for coadministration in combination therapies along with doxorubicin.

\section{Data Availability}

All other data arising from this study are contained within the article and supplementary information files.

\section{Conflicts of Interest}

The authors declare no conflicts of interest.

\section{Authors' Contributions}

Yong-Joon Jeon and Jong-Il Shin contributed equally to this work.

\section{Acknowledgments}

The natural product samples were provided by the KIST Natural Product Library, supported by the KIST Institutional Program (2Z05320). This study was supported by a grant from the Korea Health Technology R\&D Project provided to the Korea Health Industry Development Institute (KHIDI), funded by the Ministry of Health \& Welfare, Republic of Korea (Grant no. HI15C1540), along with the National Research Foundation of Korea (NRF) grant, funded by the Korean government (MSIP) (no. NRF2015R1C1A2A01053623).

\section{Supplementary Materials}

Figure S1: fraction \#3 of AGN extract markedly induced apoptosis and increased expression of CHOP in HeLa cells. (a) The MTT assay was performed for measurement of cell viability. (b) The cells were treated with $20 \mu \mathrm{g} / \mathrm{ml}$ fraction of the AGN extract for $16 \mathrm{~h}$. Immunoblot analyses were performed using specific antibodies as indicated. Figure S2: AGN extract did not enhance doxorubicin-induced apoptosis in wild type WI-38 cells. ( $\mathrm{a}$ and b) WI-38 cells were treated with the indicated concentrations of the AGN extract for $24 \mathrm{~h}$. (a) The MTT assay was performed for measurement of cell viability. (b) Immunoblot analyses were performed using specific antibodies as indicated. (c) The cells were cotreated with the indicated concentrations of doxorubicin and the AGN extract for $24 \mathrm{~h}$ and cell viability was measured using the MTT assay. (d) The cells were cotreated with 1 $\mu \mathrm{M}$ doxorubicin and $1 \mu \mathrm{g} / \mathrm{ml}$ AGN extract for the indicated time periods and subjected to immunoblot analyses using specific antibodies as indicated. Figure S3: C16 restored the AGN-mediated apoptosis regardless of the eIF $2 \alpha$-ATF4CHOP pathway. ( $a$ and $b$ ) The cells were cotreated with the indicated AGN extract and C16 for $24 \mathrm{~h}$. (a) The MTT assay was performed for measurement of cell viability. Statistical significance of the difference was calculated by Student's ttest with $* \mathrm{p}<0.01$. (b) Immunoblot analyses were performed for measurement of apoptosis using specific antibodies. (c) 
The cells were cotreated with $10 \mu \mathrm{g} / \mathrm{ml}$ AGN extract and $500 \mathrm{nM} \mathrm{C16}$ for $4 \mathrm{~h}$ (top) or $24 \mathrm{~h}$ (bottom) and immunoblot analyses were performed using specific antibodies. Figure S4: Knockdown of CHOP did not affect the apoptosis in AGN extract-treated HeLa cells. HeLa cells were transfected with EGFP- or CHOP-specific shRNA. (a) The cells were treated with the indicated concentrations of the AGN extract for $24 \mathrm{~h}$, and an MTT assay was performed to determine cell viability. (b) The cells were treated with $10 \mu \mathrm{g} / \mathrm{ml}$ AGN extract for $16 \mathrm{~h}$ and subjected to immunoblot analyses using specific antibodies as indicated. (c) The cells were cotreated with the indicated concentrations of doxorubicin and the AGN extract for $24 \mathrm{~h}$ and cell viability was measured using the MTT assay. (d) The cells were cotreated with $1 \mu \mathrm{M}$ doxorubicin and $1 \mu \mathrm{g} / \mathrm{ml} \mathrm{AGN} \mathrm{extract} \mathrm{for} \mathrm{the} \mathrm{indicated} \mathrm{time} \mathrm{periods} \mathrm{and}$ subjected to immunoblot analyses using specific antibodies as indicated. (Supplementary Materials)

\section{References}

[1] F. Arcamone, G. Cassinelli, G. Fantini et al., "Adriamycin, 14hydroxydaimomycin, a new antitumor antibiotic from $S$. peucetius var. caesius," Biotechnology and Bioengineering, vol. 11, no. 6, pp. 1101-1110, 1969.

[2] J. V. McGowan, R. Chung, A. Maulik, I. Piotrowska, J. M. Walker, and D. M. Yellon, "Anthracycline Chemotherapy and Cardiotoxicity," Cardiovascular Drugs and Therapy, vol. 31, no. 1, pp. 63-75, 2017.

[3] S. Rivankar, "An overview of doxorubicin formulations in cancer therapy," Journal of Cancer Research and Therapeutics, vol. 10, no. 4, pp. 853-858, 2014.

[4] S. M. Swain, F. S. Whaley, and M. S. Ewer, "Congestive heart failure in patients treated with doxorubicin: a retrospective analysis of three trials," Cancer, vol. 97, no. 11, pp. 2869-2879, 2003.

[5] L. Bao, A. Haque, K. Jackson et al., "Increased Expression of PGlycoprotein Is Associated with Doxorubicin Chemoresistance in the Metastatic 4T1 Breast Cancer Model," The American Journal of Pathology, vol. 178, no. 2, pp. 838-852, 2011.

[6] K. R. Hess et al., "Pharmacogenomic Predictor of Sensitivity to Preoperative Chemotherapy With Paclitaxel and Fluorouracil, Doxorubicin, and Cyclophosphamide in Breast Cancer," Journal of Clinical Oncology, vol. 24, no. 26, pp. 4236-4244, 2007.

[7] W. Hiddemann, M. Kneba, M. Dreyling et al., "Frontline therapy with rituximab added to the combination of cyclophosphamide, doxorubicin, vincristine, and prednisone (CHOP) significantly improves the outcome for patients with advancedstage follicular lymphoma compared with therapy with CHOP alone: results of a prospective randomized study of the German Low-Grade Lymphoma Study Group," Blood, vol. 106, no. 12, pp. 3725-3732, 2005.

[8] M. S. Butler, "The role of natural product chemistry in drug discovery," Journal of Natural Products, vol. 67, no. 12, pp. 2141-2153, 2004.

[9] M. Kartal, "Intellectual property protection in the natural product drug discovery, traditional herbal medicine and herbal medicinal products," Phytotherapy Research, vol. 21, no. 2, pp. 113-119, 2007.

[10] A. Ganesan, "The impact of natural products upon modern drug discovery," Current Opinion in Chemical Biology, vol. 12, no. 3, pp. 306-317, 2008.
[11] J. W. Li and J. C. Vederas, "Drug discovery and natural products: end of an era or an endless frontier?" Science, vol. 325, no. 5937, pp. 161-165, 2009.

[12] S. D. Sarker and L. Nahar, "Natural medicine: the genus Angelica," Current Medicinal Chemistry, vol. 11, no. 11, pp. 14791500, 2004.

[13] J. Zhang, L. Li, C. Jiang, C. Xing, S.-H. Kim, and J. Lü, "Anti-cancer and other bioactivities of Korean Angelica gigas Nakai (AGN) and its major pyranocoumarin compounds," Anti-Cancer Agents in Medicinal Chemistry, vol. 12, no. 10, pp. 1239-1254, 2012.

[14] C. Reddy, S. Kim, M. Hur et al., "Natural Korean Medicine Dang-Gui: Biosynthesis, Effective Extraction and Formulations of Major Active Pyranocoumarins, Their Molecular Action Mechanism in Cancer, and Other Biological Activities," Molecules, vol. 22, no. 12, p. 2170, 2017.

[15] J. H. Park, Y. J. Lee, and S. J. Keon, “Pharmacognostical studies on the Dang Gui from Korea," Korean Journal of Pharmacognosy, vol. 36, no. 2, pp. 141-144, 2005.

[16] S.-K. Cho, A. M. A. El-Aty, J.-H. Choi, M. R. Kim, and J. H. Shim, "Optimized conditions for the extraction of secondary volatile metabolites in Angelica roots by accelerated solvent extraction," Journal of Pharmaceutical and Biomedical Analysis, vol. 44, no. 5, pp. 1154-1158, 2007.

[17] M. A. Yoo, Y. K. Song, H. Jang, D. M. Kim, and S. Y. Byun, "Profiling of skin anti-aging related proteins in human dermal fibroblasts by decursin in Angelica gigas Nakai," Korean Journal of Chemical Engineering, vol. 28, no. 3, pp. 880-885, 2011.

[18] J. H. Kim, S.-J. Jeong, H.-Y. Kwon et al., “Decursin prevents cisplatin-induced apoptosis via the enhancement of antioxidant enzymes in human renal epithelial cells," Biological \& Pharmaceutical Bulletin, vol. 33, no. 8, pp. 1279-1284, 2010.

[19] L. Li, W. Li, S. Jung, Y. Lee, and Y. Kim, "Protective Effects of Decursin and Decursinol Angelate against Amyloid $\beta$-ProteinInduced Oxidative Stress in the PC12 Cell Line: The Role of Nrf2 and Antioxidant Enzymes," Bioscience, Biotechnology, and Biochemistry, vol. 75, no. 3, pp. 434-442, 2014.

[20] C. Carvalho, R. X. Santos, S. Cardoso et al., "Doxorubicin: the good, the bad and the ugly effect," Current Medicinal Chemistry, vol. 16, no. 25, pp. 3267-3285, 2009.

[21] K. Pakos-Zebrucka, I. Koryga, K. Mnich, M. Ljujic, A. Samali, and A. M. Gorman, "The integrated stress response," EMBO Reports, vol. 17, no. 10, pp. 1374-1395, 2016.

[22] N. Donnelly et al., "The eIF2alpha kinases: their structures and functions," Cellular and Molecular Life Sciences, vol. 70, no. 19, pp. 3493-3511, 2013.

[23] P. D. Lu, H. P. Harding, and D. Ron, "Translation reinitiation at alternative open reading frames regulates gene expression in an integrated stress response," The Journal of Cell Biology, vol. 167, no. 1, pp. 27-33, 2004.

[24] J. Han and R. J. Kaufman, "Physiological/pathological ramifications of transcription factors in the unfolded protein response," Genes \& Development, vol. 31, no. 14, pp. 1417-1438, 2017.

[25] J. Han, S. H. Back, J. Hur et al., "ER-stress-induced transcriptional regulation increases protein synthesis leading to cell death," Nature Cell Biology, vol. 15, no. 5, pp. 481-490, 2013.

[26] D. Hanahan and R. A. Weinberg, "Hallmarks of cancer: the next generation," Cell, vol. 144, no. 5, pp. 646-674, 2011.

[27] R. S. Y. Wong, "Apoptosis in cancer: from pathogenesis to treatment," Journal of Experimental \& Clinical Cancer Research, vol. 30, no. 1, article 87, 2011. 
[28] Y. J. Jeon et al., "Salubrinal-Mediated Upregulation of eIF2 $\alpha$ Phosphorylation Increases Doxorubicin Sensitivity in MCF7/ADR Cells," Molecules and Cells, vol. 39, no. 2, pp. 129-135, 2016.

[29] R. L. Bennett, A. L. Carruthers, T. Hui, K. R. Kerney, X. Liu, and W. S. May, "Increased Expression of the dsRNA-Activated Protein Kinase PKR in Breast Cancer Promotes Sensitivity to Doxorubicin," PLoS ONE, vol. 7, no. 9, Article ID e46040, 2012.

[30] A. C. Palmer and P. K. Sorger, "Combination Cancer Therapy Can Confer Benefit via Patient-to-Patient Variability without Drug Additivity or Synergy," Cell, vol. 171, no. 7, pp. 16781682.e13, 2017.

[31] S. Elmore, "Apoptosis: a review of programmed cell death," Toxicologic Pathology, vol. 35, no. 4, pp. 495-516, 2007.

[32] K. Sowndhararajan, P. Deepa, M. Kim, S. J. Park, and S. Kim, "A Review of the Composition of the Essential Oils and Biological Activities of Angelica Species," Scientia Pharmaceutica, vol. 85, no. 3, p. 33, 2017.

[33] J. Couturier, M. Morel, R. Pontcharraud et al., “ Interaction of Double-stranded RNA-dependent Protein Kinase (PKR) with the Death Receptor Signaling Pathway in Amyloid $\beta(\mathrm{A} \beta)$ treated Cells and in APP ," The Journal of Biological Chemistry, vol. 285, no. 2, pp. 1272-1282, 2010.

[34] J. Couturier, M. Paccalin, M. Morel et al., "Prevention of the $\beta$-amyloid peptide-induced inflammatory process by inhibition of double-stranded RNA-dependent protein kinase in primary murine mixed co-cultures," Journal of Neuroinflammation, vol. 8, no. 1, p. 72, 2011.

[35] C.-H. Yoon, E.-S. Lee, D.-S. Lim, and Y.-S. Bae, "PKR, a p53 target gene, plays a crucial role in the tumor-suppressor function of p53," Proceedings of the National Acadamy of Sciences of the United States of America, vol. 106, no. 19, pp. 7852-7857, 2009.

[36] S. J. Kim, K. M. Park, N. Kim, and Y. I. Yeom, "Doxorubicin prevents endoplasmic reticulum stress-induced apoptosis," Biochemical and Biophysical Research Communications, vol. 339, no. 2, pp. 463-468, 2006.

[37] C. Hetz, "The unfolded protein response: controlling cell fate decisions under ER stress and beyond," Nature Reviews Molecular Cell Biology, vol. 13, no. 2, pp. 89-102, 2012.

[38] G. Qing, B. Li, A. Vu et al., "ATF4 Regulates MYC-Mediated Neuroblastoma Cell Death upon Glutamine Deprivation," Cancer Cell, vol. 22, no. 5, pp. 631-644, 2012.

[39] J. L. Armstrong, R. Flockhart, G. J. Veal, P. E. Lovat, and C. P. F. Redfern, "Regulation of endoplasmic reticulum stress-induced cell death by ATF4 in neuroectodermal tumor cells," The Journal of Biological Chemistry, vol. 285, no. 9, pp. 6091-6100, 2010.

[40] Y. Li, Y. Guo, J. Tang, J. Jiang, and Z. Chen, "New insights into the roles of CHOP-induced apoptosis in ER stress," Acta Biochimica et Biophysica Sinica, vol. 46, no. 8, pp. 629-640, 2014.

[41] Z. Xu, Y. Bu, N. Chitnis, C. Koumenis, S. Y. Fuchs, and J. A. Diehl, "miR-216b regulation of c-Jun mediates GADD153/ CHOP-dependent apoptosis," Nature Communications, vol. 7, p. 11422, 2016.

[42] A. R. Safa and K. E. Pollok, "Targeting the anti-apoptotic protein c-FLIP for cancer therapy," Cancers, vol. 3, no. 2, pp. 1639-1671, 2011.

[43] N.-H. Yim, J. H. Lee, W.-K. Cho, M. C. Yang, D. H. Kwak, and J. Y. Ma, "Decursin and decursinol angelate from Angelica gigas Nakai induce apoptosis via induction of TRAIL expression on cervical cancer cells," European Journal of Integrative Medicine, vol. 3, no. 4, pp. e293-e301, 2011.
[44] K. D. McCullough, J. L. Martindale, L. O. Klotz, T. Y. Aw, and N. J. Holbrook, "Gadd153 sensitizes cells to endoplasmic reticulum stress by down-regulating $\mathrm{Bc} 12$ and perturbing the cellular redox state," Molecular and Cellular Biology, vol. 21, no. 4, pp. 1249-1259, 2001.

[45] T. Gotoh, K. Terada, S. Oyadomari, and M. Mori, "hsp70DnaJ chaperone pair prevents nitric oxide- and CHOP-induced apoptosis by inhibiting translocation of Bax to mitochondria," Cell Death \& Differentiation, vol. 11, no. 4, pp. 390-402, 2004.

[46] Y. Jiang, J. Piao, H.-J. Cho, W.-S. Kang, and H.-Y. Kim, "Improvement in antiproliferative activity of Angelica gigas Nakai by solid dispersion formation via hot-melt extrusion and induction of cell cycle arrest and apoptosis in HeLa cells," Bioscience, Biotechnology, and Biochemistry, vol. 79, no. 10, pp. 1635-1643, 2015.

[47] J. Jang, S.-J. Jeong, H.-Y. Kwon et al., “Decursin and Doxorubicin Are in Synergy for the Induction of Apoptosis via STAT3 and/or mTOR Pathways in Human Multiple Myeloma Cells," Evidence-Based Complementary and Alternative Medicine, vol. 2013, Article ID 506324, 13 pages, 2013.

[48] H. S. Choi, S.-G. Cho, M. K. Kim et al., "Decursin in Angelica gigas Nakai (AGN) Enhances Doxorubicin Chemosensitivity in NCI/ADR-RES Ovarian Cancer Cells via Inhibition of Pglycoprotein Expression," Phytotherapy Research, vol. 30, no. 12, pp. 2020-2026, 2016.

[49] J. Kim, M. Yun, E.-O. Kim et al., "Decursin enhances TRAILinduced apoptosis through oxidative stress mediated- endoplasmic reticulum stress signalling in non-small cell lung cancers," British Journal of Pharmacology, vol. 173, no. 6, pp. 1033-1044, 2016.

[50] S. H. Son, M.-J. Kim, W.-Y. Chung et al., "Decursin and decursinol inhibit VEGF-induced angiogenesis by blocking the activation of extracellular signal-regulated kinase and c-Jun Nterminal kinase," Cancer Letters, vol. 280, no. 1, pp. 86-92, 2009.

[51] S. H. Son, K.-K. Park, S. K. Park et al., "Decursin and decursinol from Angelica gigas inhibit the lung metastasis of murine colon carcinoma," Phytotherapy Research, vol. 25, no. 7, pp. 959-964, 2011. 

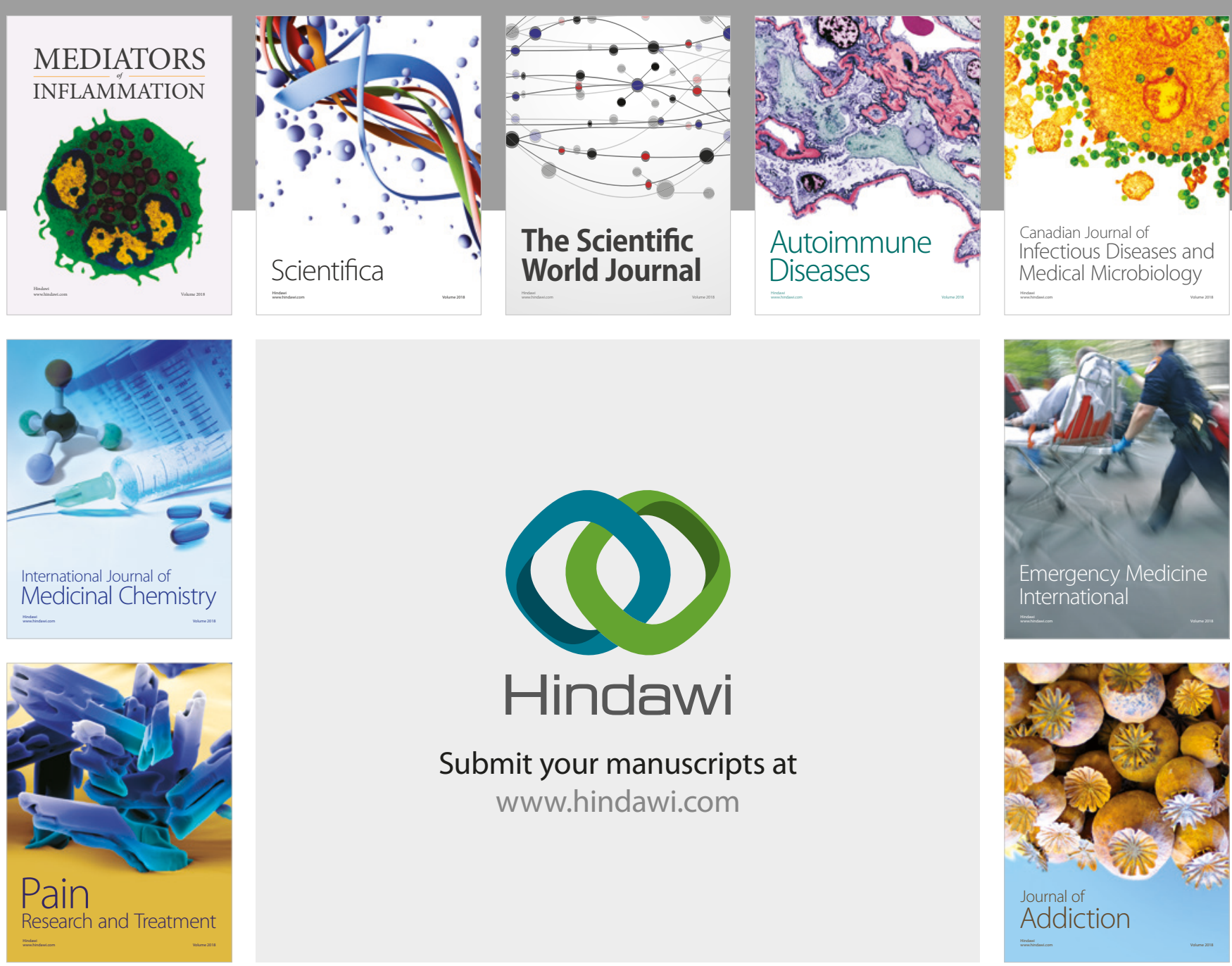

Canadian Journal of
Infectious Diseases and Medical Microbiology

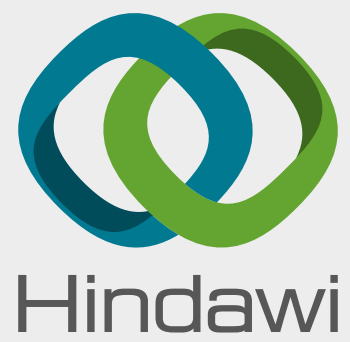

Submit your manuscripts at

www.hindawi.com
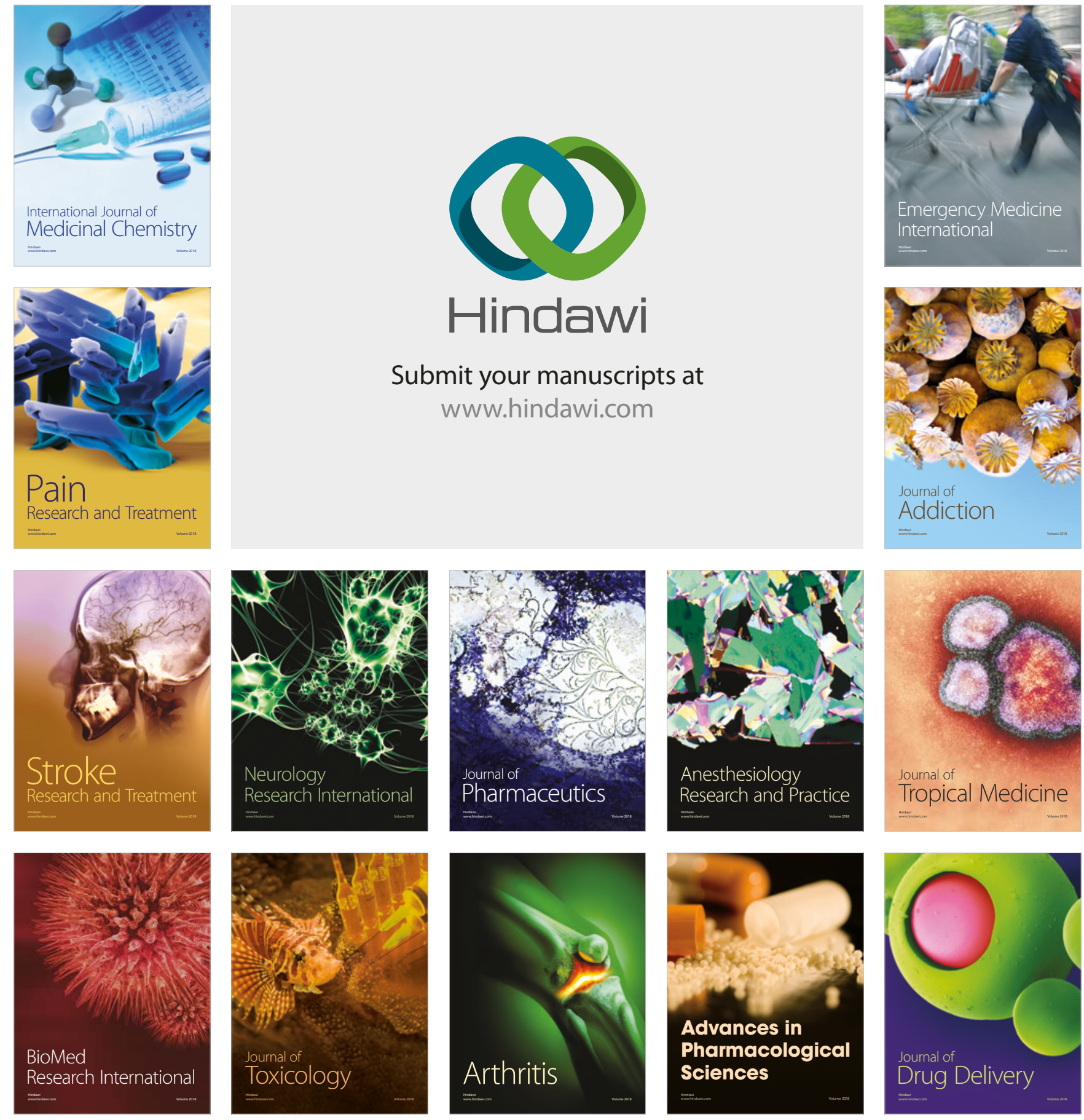